\title{
EDITORIAL
}

\section{La resistencia antimicrobiana del Streptococcus pneumoniae como un problema de salud pública}

D esde su descubrimiento, los fármacos antimimicrobianos han probado, en forma contundente, que son efectivos para el control de las infecciones bacterianas; sin embargo, pronto se hizo evidente que los patógenos bacterianos no eran propensos a rendirse incondicionalmente, ya que algunos de ellos rápidamente mostraron la emergencia y diseminación de resistencia hacia diversos antimicrobianos, al poco tiempo de que éstos fueron introducidos para uso en humanos. ${ }^{1,2}$

En el caso del neumococo -un importante agente infeccioso causante de otitis media, neumonía y septicemia, especialmente en población pediátrica- se sabe que hasta antes de 1960 todos los aislamientos del organismo eran exquisitamente susceptibles a la penicilina, al determinar la concentración mínima inhibitoria (CMI) in vitro del antibiótico $(\mathrm{CMI}<0.006 \mu \mathrm{g} / \mathrm{ml})$. En México, las cepas de Streptococcus pneumoniae resistentes eran relativamente raras hasta antes de 1993, ${ }^{3-5}$ y la penicilina fue el fármaco de primera elección para el tratamiento empírico de las infecciones neumocócicas por más de 50 años.

La presencia de cepas de S. pneumoniae con susceptibilidad disminuida a la penicilina y otros agentes antimicrobianos es ahora un hecho reconocido en el ámbito mundial, lo que plantea situaciones que deben ser reanalizadas en función de ofrecer alternativas para el tratamiento y la prevención de este agente oportunista.

Desde la década que va de 1967 a 1977, en varias partes del mundo se publicaron informes esporádicos de aislamientos de neumococo resistente a la penicilina. Sin embargo, el evento epidemiológico más dramático fue la epidemia de enfermedad neumocócica causada por cepas multirresistentes en los hospitales sudafricanos (1977), durante la cual los aislamientos demostraron CMI muy elevadas. ${ }^{6,7}$

En cuanto a la forma en que el neumococo expresa la resistencia a la penicilina, se ha precisado que la misma obedece a alteraciones estructurales de la pared celular bacteriana. Así, se ha identificado que, cuando menos cuatro de las cinco proteínas enlazadoras de penicilina (PBP, por sus siglas en inglés), sufren cambios dramáticos en su afinidad para unirse a las moléculas del antibiótico. Estas alteraciones son debidas a mutaciones genéticas estables mediadas por el cromosoma, respecto a las cuales, hasta ahora, no se ha demostrado que estén ligadas al aumento o a la disminución de la virulencia del S. pneumoniae. ${ }^{8}$ Un dato epidemiológico importante es que la presencia de CMI elevadas, es un marcador de la resistencia múltiple a diversas especies de antimicrobianos como el cloranfenicol, el trimetoprim-sulfametoxazol, la eritromicina, la tetraciclina y los aminoglucósidos.

En consecuencia, la resistencia del neumococo ha quedado claramente establecida como un problema de salud pública durante las dos últimas décadas, y cabe añadir que de la epidemia de la enfermedad neumocócica se ha aprendido que por el uso excesivo de antimicrobianos, se seleccionan clonas que se diseminan en forma continental. Esto es particularmente cierto por el uso indiscriminado y empírico de antimicrobianos para tratar las infecciones respiratorias (la mayoría de etiología viral) y la otitis media aguda, en especial por la administración de cefalosporinas y macrólidos por vía oral.

En Estados Unidos de América, por ejemplo, la proporción de cepas altamente resistentes a la penicilina aumentó dramáticamente: entre 1979 y 1987 era de $0.02 \%$; entre 1991 y 1992, de $1.3 \%$; entre 1993 y 1994, de 3.2\%, y en el periodo 1994-1995 llegó hasta $9.5 \% .{ }^{9}$

El panorama epidemiológico de resistencia para otros antimicrobianos es igualmente preocupante ${ }_{1}^{10}$ ya que usualmente se les emplea para tratar infecciones neumocócicas. Así, en algunos casos selectos se tienen los siguientes porcentajes de resistencia: amoxicilina, $18.1 \%$; cefadroxil, $91.7 \%$; cefaclor, $38.3 \%$; cefuroxima, $19.5 \%$; cefpodoxina, $18.6 \%$; cefixima, 
27.8\%; cefotaxima, $4.0 \%$; cefepima, $8.2 \%$; TMP/SMX, $19.8 \%$; tetraciclina, $10.2 \%$; clindamicina, $3.7 \%$; cloranfenicol, 3.9\%; macrólidos, 11.7-14.3\%, y vancomicina, prácticamente sin resistencia. ${ }^{11}$

Los datos presentados envían un mensaje inequívoco: la rapidez con la que evoluciona la resistencia del S. pneumoniae, complica la selección de antimicrobianos para el tratamiento de las infecciones neumocócicas, fundamentalmente de las diseminadas.

En México la información es parcial; en realidad la procedencia de más de $80 \%$ de las cepas recuperadas corresponden al Hospital Infantil de México Federico Gómez, ${ }^{4,5,12,13}$ por lo cual la situación epidemiológica de la resistencia del neumococo debe considerarse con esa gran limitante. La razón fundamental de tal carencia es que en el ámbito nacional no se hace un esfuerzo intencionado para recuperar las cepas de diferentes fuentes.

A pesar de que existe un bajo rendimiento de recuperación de cepas, es posible reconocer que, de 1993 a 1998, la resistencia bacteriana múltiple a diversos antimicrobianos mostró una tendencia progresivamente creciente, fenómeno semejante al que se notifica en la literatura sobre el tema. Este hecho obliga a la implementación de programas de vigilancia epidemiológica que permitan prevenir la emergencia de cepas de S. pneumoniae, mediante el uso prudente de antimicrobianos.

Respecto a la composición capsular del neumococo, se ha observado, prácticamente en todo el mundo, que cinco serotipos $(6,9,14,19$ y 23$)$ son los que con más frecuencia exhiben resistencia a la penicilina y multirresistencia a otros antimicrobianos. La proporción de esos serotipos en la comunidad hace evidente el problema de resistencia de esta bacteria.

Por ahora, son tres las acciones que se deben llevar a cabo: la primera y más urgente sería establecer el manejo terapéutico de las infecciones neumocócicas; ${ }^{14}$ la segunda sería establecer los criterios clínicos que permitan con cierta eficacia definir a los pacientes con factores de riesgo de adquirir la infección neumocócica, así como reconocer a aquellos que pudiesen tener un curso clínico diferente al de la población general y que tengan un riesgo importante de perder la vida; finalmente, la tercera sería diseñar, con cierta precisión, la fórmula para conformar la vacuna conjugada con los serotipos prevalentes en infecciones sistémicas. ${ }^{12,15}$

Dentro de este contexto, en este número de Salud Pública de México se incluye un trabajo realizado, durante los años recientes, en el Hospital Infantil de México Federico Gómez, ${ }^{16}$ correspondiente al programa permanente que esta institución ha puesto en marcha para la vigilancia clínica, microbiológica y epidemiológica de las infecciones invasivas provocadas por el S. pneumoniae en la población ahí atendida. Los resultados que se notifican ofrecen datos de actualidad y de interés médico, y si bien se circunscriben a una casuística aislada, reiteran la necesidad de ampliar el conocimiento en México de este problema de salud pública.

Dr. Ernesto Calderón Jaimes*

\section{Referencias}

1. Calderón JE. Resistencia a los fármacos antimicrobianos. En: Espinosa $L R$, ed.Terapéutica en enfermedades infecciosas. 2a. edición. México, D.F.: Impresora y Litografía U niversal, 1999:23-40.

2.0 'BrienTF.The global epidemic of antimicrobial resistance and the need to monitor and manage it locally. C lin Infect D is 1997;24 suppl 1:2-8.

3. Guiscafré GH, García MM, Trejo PA, García M, Hernández VR, Muñoz $H O$. Frecuencia de Haemophilus influenzae resistente a ampicilina y de Streptococcus pneumoniae resistente a penicilina en portadores sanos.A rch Invest Med Mex 1981;12:141-151.

4. Calderón JE, Conde-González CJ, Gatica MR, Rivera SR, Hernando N, Barriga AG et al. Antimicrobial resistance among bacterial isolates from children. Curr Ther Res 1993; 54:482-491.

5. Calderón JE, Echániz AG, Conde-G onzález CJ, Rivera SR, Barriga AG, Solórzano SF et al. Resistencia y serotipificación de 83 cepas de Streptococcus pneumoniae aisladas de niños portadores asintomáticos y enfermos. Bol Med Hosp Infant Mex 1993:50:854-860.

6. Tomasz A. Antibiotic resistance in Streptococcus pneumoniae. Clin Infect D is 1997;24 suppl 1:385-388.

7. Klugman KP, Koornhof HJ. D rug resistance patterns and serogroups or serotypes of pneumococcal isolates from cerebrospinal fluid or blood, 1979-1986.J Infect D is 1988;158:956-964.

8. Tomasz A. The pneumococcus at the gates. N Engl J Med 1995;333: 514-515.

9. Doern GS, Brueggemann A, Holley HP, Rauch AM. Antimicrobial resistance of Streptococcus pneumoniae recovered from outpatients in the United States during the winter months of 1994 to 1995: Results of a 30 center national surveillance study. Antimicrob Agents Chemother 1996; 40:1208-1213.

10. Doern GV, Pfaller MA, Kugler K, Freeman J, Jones RN. Prevalence of antimicrobial resistance among respiratory tract isolates of Streptococcus pneumoniae in N orth A merica: 1997 results from the SEN TRY.Antimicrobial Surveillance Program. Clin Infect D is 1998;27:764-770.

11. Mufson MA. Editorial response: Penicillin-resistance Streptococcus pneumoniae increasingly threatens the patient and challenges the physician. C lin Infect $D$ is 1998;27:771-773.

12. Sniadack DH, Schwartz B, Lipman H, Bogaertz J, Butler JC, Dagan R et al. Potential interventions for the prevention of childhood pneumonia: Geographic and temporal differences in serotype and serogroup distribution of sterile site pneumococcal isolates from children-implications for vaccine strategies. Pediatr Infect D is J 1995;14:503-510.

13. Echániz AG, Velázquez ME, C arnalla BN , Soto N A, Solórzano SF, Pérez MA et al.Antimicrobial susceptibilities and capsular types of invasive Streptococcus pneumoniae isolated in children in Mexico City. Microb D rug Resist 1997;3:153-157.

14. Kaplan SL, Mason EO. Management of infections due to antibioticresistant Streptococcus pneumoniae. Clin Microbiol Rev 1998;11:628-644. 15. De Velasco EA,Verheul FM, Verhoef J, Snippe H. Streptococcus pneumoniae:Virulence factors, pathogenesis and vaccines. Microbiol Rev 1995;59: 591-603.

16. Gómez-Barreto D, Calderón-Jaimes E, Rodríguez RS, Espinosa de los Monteros LE, Juárez M. Características clínico-microbiológicas de la meningitis por Streptococcus pneumoniae resistente a la penicilina. Salud Publica Mex 1999;41:397-404.

* División de Medicina Experimental, Instituto Nacional de Pediatría, México 